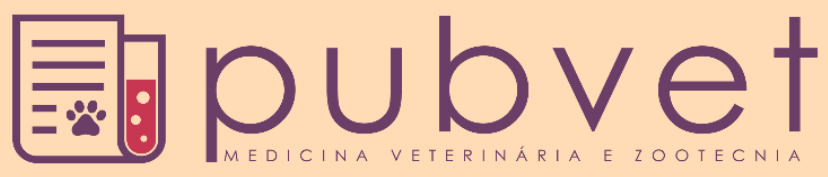

https://doi.org/10.31533/pubvet.v15n07a854.1-4

\title{
Laceração perineal em ovelha da raça Texel: Relato de caso
}

\author{
Carla Fredrichsen Moya $^{1 *}$, Caroline Barabach $^{2} \bullet$, Heloisa Godoi Bertagnon $^{3 \bullet} \mathbb{E}$ \\ ${ }^{I}$ Professora da Universidade Estadual do Centro-Oeste, Departamento de Medicina Veterinária, Guarapuava-PR, Brasil \\ ${ }^{2}$ Médica Veterinária Autônoma, Guarapuava - PR, Brasil. \\ ${ }^{3}$ Professora da Universidade Estadual do Centro-Oeste, Departamento de Medicina Veterinária, Guarapuava-PR, Brasil. \\ *Autor para correspondência, E-mail: carlafredrichsen@yahoo.com.br
}

Resumo. A laceração perineal é uma das lesões mais comuns relacionadas ao parto, principalmente na espécie equina e, em menor frequência, na espécie bovina. Esta doença é incomum em ovinos, justificando a importância deste relato de caso. O presente trabalho teve como objetivo descrever um caso clínico de laceração perineal de terceiro grau em uma ovelha. Foi atendida na Escola de Clínica Veterinária da UNICENTRO, uma ovelha Texel, primípara, com 1,5 anos de idade, com histórico de laceração durante o nascimento de cordeiro único com 4,5 kg. Ao exame físico, todos os parâmetros estavam dentro da normalidade para a espécie. Na avaliação da região perineal, a laceração envolvia a comissura dorsal da vulva, esfíncter anal e uma pequena porção do vestíbulo. O tratamento preconizado foi a intervenção cirúrgica imediata, uma vez que, o animal foi atendido prontamente após o parto, e ainda não havia desenvolvido processo inflamatório local intenso. Foram realizadas a anestesia epidural baixa e local com lidocaína 2\%. A sutura da mucosa vestíbulo vaginal foi realizada no padrão Cushing, usando fio absorvível n.0. Para a reconstrução vulvar foi necessária a ressecção de um segmento da junção mucocutânea de cada lábio vulvar, seguida de uma sutura em padrão ponto simples separado, utilizando um fio inabsorvível n.2-0. No pós-operatório, foi administrado antibiótico a base de sulfa+ trimetoprim $(15 \mathrm{mg} / \mathrm{kg}$, IM, SID), durante sete dias consecutivos e flunexin meglumine $(1,1$ $\mathrm{mg} / \mathrm{kg}$, IM, SID), por três dias consecutivos. O curativo local foi realizado a cada 12 horas empregando-se iodo povidine diluído em solução de $\mathrm{NaCl} 0,9 \%$ e pomada repelente na região perineal, até a remoção dos pontos. A sutura externa foi removida 10 dias após a cirurgia, com completa cicatrização da ferida. Apesar do sucesso cirúrgico, o proprietário optou por remover o animal da reprodução após desmame do cordeiro.

Palavras chave: Laceração perineal, ovino, parto

\section{Perineal laceration in a Texel breed ewe: Case report}

Abstract. The perineal laceration is one of the most common injuries related to the
parturition, mostly in the equine specie, and, less frequently, in the bovine species. This
disease is uncommon in ovine, therefore justifying the importance this case report. The
present work aimed to present a case report of a third-degree laceration in a Texel breed
ewe. Where received at the Veterinary Clinics School of the UNICENTRO, a Texel ewe,
primiparous, 1.5 year of age, with historic of laceration during the birth of an only male
lamb with $4,5 \mathrm{~kg}$. During the physical examination, all parameters were normal, but the
laceration involved the dorsal commissure of the vulva, anal sphincter and a small portion
of the vestibule. The preconized treatment was the immediate surgical intervention, once
the animal was recent lambed and still not yet developed an intense local edema. Due the
lesion at rectum and vestibule, were performed an epidural and infiltrative local anesthesia
with lidocaine $2 \%$. The vaginal vestibular mucosa suture pattern was Cushing, using an
absorbable thread n.0. To the vulva reconstruction was necessary the resection of a segment 
of the mucous-cutaneous junction of each vulvar lip, followed by a single point suture, using a non-absorbable thread n.2-0. At the postoperative were administered sulfa + trimetoprim $(15 \mathrm{mg} / \mathrm{kg}$, IM, SID) during seven days, and flunixin meglumine $(1.1 \mathrm{mg} / \mathrm{kg}$, IM, SID) for three days. Daily wound cleaning was performed using povidine iodine diluted in $\mathrm{NaCl} 0.9 \%$ solution, finishing with a fly repellent around the perineal region. The suture was removed after 10 days, with the complete wound healing. Despite the successful surgery treatment outcome, the owner decided to discard the ewe after the lamb weaning.

Keywords: Perineal laceration, ovine, parturition

\section{Introdução}

A laceração perineal é uma das lesões mais comuns relacionadas ao parto, principalmente na espécie equina, sendo classificada em primeiro, segundo e terceiro grau de acordo com a extensão da lesão tecidual (Noakes et al., 2001). As causas da ocorrência das lacerações são relacionadas a problemas no parto, como resultado de tração forçada, erros de estática fetal, desproporção materno-fetal, principalmente em fêmeas primíparas (Dreyfuss et al., 1990).

Em relação a classificação, a laceração de primeiro grau envolve apenas a mucosa do vestíbulo e a pele da comissura dorsal da vulva. O segundo grau inclui a mucosa e a submucosa da vulva dorsal e parte da musculatura do corpo perineal, em particular o músculo constritor da vulva. Não há dano à mucosa retal. O terceiro grau resulta em ruptura da parede vestibular e, às vezes, vaginal, e ruptura do corpo perineal, esfíncter anal e parede retal. Como consequência, existe uma abertura comum entre o reto e o vestíbulo. A preocupação é que a perda da conformação vulvar e vestibular funcional da abóbada vaginal eventualmente pode evoluir para um quadro de infertilidade por maior propensão à infecção uterina (El-Seddawy, 1993; Farag et al., 2000; Noakes et al., 2001; Saleh et al., 1988).

A laceração de primeiro grau raramente interfere na fertilidade subsequente da fêmea, a menos que um processo inflamatório/infeccioso seja instalado e seguido por aderências das superfícies da vagina. Este efeito de aderências pode provocar uma estenose ou bloqueio do lúmen vaginal. As aderências geralmente podem ser desfeitas manualmente, com a administração de pomada a base de antissépticos para reduzir a recorrência das mesmas. O prognóstico é reservado, em casos em que ocorre a oclusão completa. Em relação a laceração de segundo grau, deve-se empregar no pós-parto imediato cuidados adequados para correta cicatrização da ferida, incluindo antibiótio tópico e/ou sistêmico, essas lesões raramente causam infertilidade (Hudson, 1972).

Contudo, em lesões de terceiro grau é recomendada a correção cirúrgica, cujo objetivo é refazer uma parede funcional entre o reto e a vagina ou vestíbulo vaginal. Assim, a reconstrução da laceração perineal nesse caso faz-se necessária para o retorno do animal à reprodução. A cirurgia é feita com o animal em estação, sendo necessária a realização de uma sedação do animal (xilazina), seguida de anestesia epidural lombosacra ou sacrococcígea com lidocaína $2 \%$. O reto deve ser esvaziado manualmente. Neste momento, um exame da genitália interna pode ser realizado com maior precisão para avaliação da extensão das lesões. O prognóstico é reservado, em função do grau de comprometimento da mucosa retal e vaginal e desenvolvimento de infecção uterina (El Maghraby, 2002; LeBlanc, 1999; Woodie, 2006). No pós-operatório emprega-se antibiótico sistêmico de amplo espectro, associado a curativo tópico com solução antisséptica (Hudson, 1972).

Esta enfermidade é incomum em ovinos, sendo mais frequente em equinos, e de menor ocorrência na espécie bovina, justificando a importância deste relato de caso. Frente ao exposto anteriormente, o presente estudo tem por objetivo relatar um caso raro de laceração peineal de terceiro grau em uma ovelha, da raça Texel, atendida na Clínica Escola Veterinária da UNICENTRO.

\section{Relato de caso}

Foi atendida na Escola de Clínica Veterinária (Cevet) da UNICENTRO, uma ovelha, da raça Texel, primípara, com 1,5 anos de idade, com histórico de laceração durante o nascimento de cordeiro único, pesando 4,5 kg. Ao exame físico, observou-se que os parâmetros clínicos (FC, FR, TPC, ToC) estavam dentro do padrão de normalidade para a espécie. Contudo, na avaliação da região perineal, notou-se que 
a laceração envolvia a comissura dorsal da vulva, esfíncter anal e uma pequena porção da mucosa vestíbulo vaginal (Figura 1A).

Mediante os achados clínicos, o tratamento preconizado foi a intervenção cirúrgica imediata, uma vez que, o animal foi atendido prontamente após o parto, e ainda não havia desenvolvido processo inflamatório local intenso.

No pré-cirúrgico, o animal foi sedado, com xilazina $(0,05 \mathrm{mg} / \mathrm{kg}$, IM), em seguida procedeu-se a tricotomia da região sacrococcígea e a antissepsia com iodo $2 \%$ e álcool $70 \%$, para posterior realização da anestesia epidural sacrococcígea com lidocaína $2 \%(0,1 \mathrm{~mL} / \mathrm{kg})$, sem vasoconstritor. Na sequência, a lavagem da região perineal com água e detergente neutro, seguida de antissepsia com solução aquosa de clorexidina $2 \%$ (não alcoólica). Após esse procedimento realizou-se a anestesia local com a administração de $5 \mathrm{~mL}$ de lidocaína $2 \%$, na região subcutânea dos lábios vulvares.

Optou-se por fazer a correção cirúrgica, em uma única etapa, em função da pequena extensão da lesão. Primeiramente, foi realizada a sutura da mucosa vestíbulo vaginal em padrão Cushing, com fio absorvível n.0 (poliglactina 910). Na sequência, para a reconstrução vulvar foi necessária a ressecção de um segmento da junção mucocutânea de cada lábio vulvar com cerca de $3 \mathrm{~mm}$ de largura, seguida de uma sutura em padrão ponto simples separado, utilizando um fio inabsorvível n.2-0 (náilon monofilamento), conforme observado na Figura 1B.

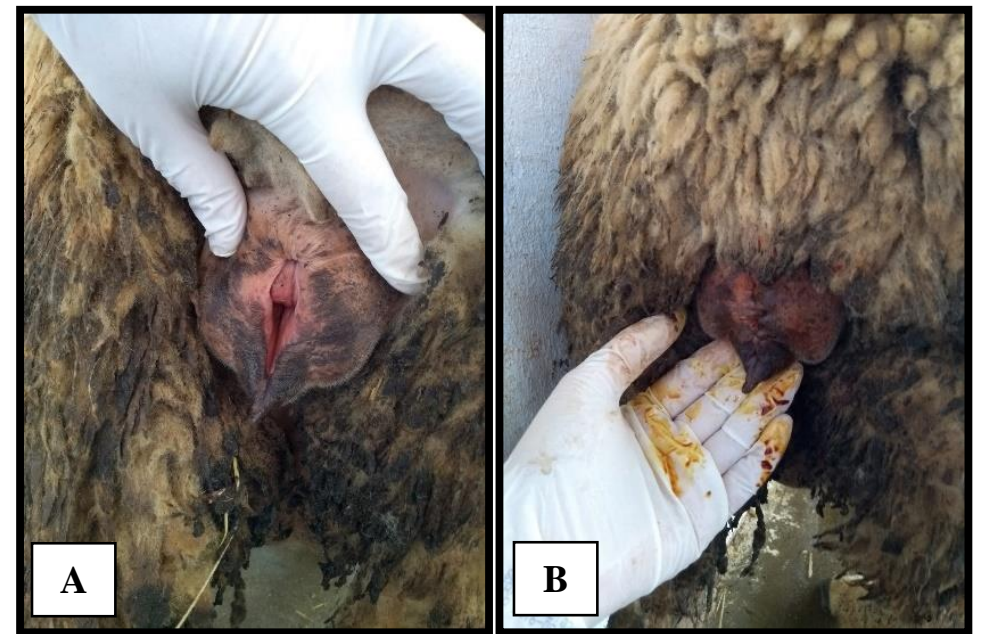

Figura 1. Região perineal de uma ovelha da raça Texel. (A) destaque para laceração de terceiro grau com comprometimento do esfíncter anal; (B) após realização da correção cirúrgica da laceração.

No pós-operatório foram administrados sulfa + trimetoprim $(15 \mathrm{mg} / \mathrm{kg}$, IM, a cada 24 horas $)$, durante sete dias consecutivos e flunixin meglumine $(1,1 \mathrm{mg} / \mathrm{kg}$, IM, a cada 24 horas), por três dias consecutivos. O curativo tópico foi realizado, a cada 12 horas, empregando-se iodo povidine diluído em solução de $\mathrm{NaCl}$ 0,9\% para limpeza da ferida cirúrgica, associado a uma pomada repelente e larvicida na região perineal, até a remoção dos pontos. A sutura externa foi removida dez dias após a cirurgia, com completa cicatrização da ferida.

\section{Discussão}

A laceração de terceiro grau observada no caso atendido está de acordo com o descrito na literatura, pois havia a ruptura da comissura dorsal da vulva, do corpo perineal e comprometimento do esfíncter anal, formando uma abertura comum entre o reto e o vestíbulo (Cofrades et al., 2004; El-Seddawy, 1993; Farag et al., 2000).

A etiologia provável para essa lesão pode estar relacionada a fêmea ser primípara e o cordeiro ter nascido com 4,5 kg, corroborando com o descrito na literatura que cita a desproporção materno-fetal, principalmente em fêmeas primíparas como causa de lacerações (Dreyfuss et al., 1990; Noakes et al., 2001). 
O tratamento preconizado nesse relato foi a correção cirúrgica, em uma única etapa, indicada para os casos de laceração perineal de terceiro grau (LeBlanc, 1999; Woodie, 2006). Nesse caso, a realização da sedação do animal e da anestesia epidural são indicadas (El Maghraby, 2002), o que vai ao encontro do procedimento feito nesse estudo, em que se empregou a sedação com xilazina e a epidural com lidocaína $2 \%$, associada a anestesia local.

No pós-operatório optou-se pelo uso de antibiótico de amplo espectro e curativo tópico, para combater possível quadro infeccioso, conforme o descrito na literatura (Hudson, 1972), além da administração de anti-inflamatório não esteroidal, para auxiliar no controle do processo inflamatório local.

\section{Considerações finais}

O diagnóstico precoce e a intervenção adequada são imprescindíveis para a correção da laceração perineal. Nesse relato, a correção cirúrgica imediata mostrou-se eficiente, com completa cicatrização da região afetada, sem complicações no pós-operatório imediato. Apesar do sucesso cirúrgico, o proprietário optou por remover o animal da reprodução após desmame do cordeiro.

\section{Referências}

Cofrades, S., Serrano, A., Ayo, J., Solas, M. T., Carballo, J., \& Jiménez Colmenero, F. (2004). Restructured beef with different proportions of walnut as affected by meat particle size. European Food Research and Technology, 218(3), 230-236. https://doi.org/10.1007/s00217-003-0808-y

Dreyfuss, D. J., Tulleners, E. P., Donawick, W. J., \& Ducharme, N. G. (1990). Third-degree perineal lacerations and rectovestibular fistulae in cattle: 20 cases (1981-1988). Journal of the American Veterinary Medical Association, 196(5), 768-770.

El-Seddawy, F. D. (1993). Surgical repair of perineal lacerations in mares and Friesian cows. Journal of Egyptian Veterinary Medical Association, 534, 681-691.

El Maghraby, H. M. (2002). A retrospective study on some surgical affections of the perineum and vagina in farm animals. Zagazig Veterinary Journal, 30(30), 84-100.

Farag, K. A., Berbish, E. A., \& Ghoneim, I. M. (2000). A one-stage repair of third-degree rectovestibular lacerations in the mare: an experimentaland clinical study. Journal of Egyptian Veterinary Medical Association, 60(2), 143-151.

Hudson, R. S. (1972). Repair of perineal lacerations in the cow. The Bovine Practitioner, 7, 34-40.

LeBlanc, M. M. (1999). Diseases of the vagina, vestibule and vulva. In P. T. Colahan, A. M. Merritt, \& J. N. Moore (Eds.), Equine medicne and surgey (pp. 1175-1193).

Noakes, D. E., Parkinson, T. J., \& England, G. C. W. (2001). Arthur's Veterinary Reproduction. Elsevier.

Saleh, M. S., Gohar, H. M., El-Keiey, M. T., Ibrahim, I. M., \& Abdel-Hamid, M. A. (1988). Reconstruction of a third-degree rectovestibular laceration in an Arabian mare. Journal of Egyptian Veterinary Medical Association, 48(4), 629-635.

Woodie, B. (2006). The vulva vestibule, vagina and cervix. In J. A. Auer \& J. A. Strick (Eds.), Equine surgey (pp. 845-852). Sauders Elsevier.

Histórico do artigo:

Recebido: 24 de fevereiro de 2021

Aprovado: 23 de março de 2021.
Licenciamento: Este artigo é publicado na modalidade Acesso Aberto sob a licença Creative Commons Atribuição 4.0 (CC-BY 4.0), a qual permite uso irrestrito, distribuição, reprodução em qualquer meio, desde que $\mathrm{o}$ autor e a fonte sejam devidamente creditados. 